\title{
Structural adjustment and endogenous worker recall probabilities*
}

\author{
Frode Meland ${ }^{\dagger}$ and Gaute Torsvik ${ }^{\dagger}$ \\ University of Bergen
}

March 14, 2002

\begin{abstract}
In this paper we investigate the incentives of unemployed workers to wait for a recall when recall probabilities are endogenously determined by the waiting decisions of others. Because of a positive externality that arise when workers seek new employment, an excessive number of workers choose to wait for a recall, and structural adjustment is slow. We also find that a small reduction in the unemployment benefits, or introducing a small cash bonus for workers that get a new job, may have no effect on unemployment in some cases, while eradicating significant levels of unemployment in other cases. Our analysis suggests that the government may face a Samaritan's Dilemma if it can influence the recall probability of workers, and that multiple equilibria may exist in a game involving both workers and an unemployment-averse government. Furthermore, we explore a link to the war of attrition literature, showing that the Bulow and Klemperer (1999) "one too many"-result may not hold if there is uncertainty concerning when the game ends.

Keywords: structural adjustment, unemployment, recalls, search, war of attrition

JEL classification: D83, J64, R23
\end{abstract}

*We thank Sjur D. Flåm, Odd Godal, Kjell Erik Lommerud and seminar participants at the University of Bergen for valuable suggestions and advice.

${ }^{\dagger}$ Both: University of Bergen, Fosswinckelsgt. 6, N-5007 Bergen, Norway. E-mail: frode.meland@econ.uib.no and gaute.torsvik@econ.uib.no. 


\section{Introduction}

Consider an economy in which a sector, or a region, experiences a slump in demand. Many workers loose their job. Each unemployed worker can choose either to stay and wait for better times, or undertake a costly move and search for a job in another sector. What will she do, stay or move?

As usual, the answer depends on many factors - such as the magnitude of the moving costs, the replacement ratio of the unemployment benefits, on the availability of jobs elsewhere and on the prospect of getting rehired in the old sector. The last factor, the prospect of getting recalled to a job - an alternative that does not require costly search - is usually not considered in search models. As Katz (1986) notes, the standard job search model focuses on the search behavior of new entrants to the labor force, or workers' permanently displaced from their previous employers. By not paying attention to recall possibilities, the models overlook a highly relevant factor.

Meyer and Katz (1990) show that in their sample $70 \%$ of the unemployed workers that received unemployment benefits, expected to be recalled to previous work. They also find that the prospect of a recall influences job search behavior, reemployment earnings and the duration of unemployment spells. Katz (1986) extends the standard job search model by including an exogenous recall probability. He shows that an improvement in the recall prospects reduces the probability of getting a new job both by increasing the reservation wage, and by lowering the intensity of search. Pissarides (1982) is another job search model that takes the prospect of recalls into account.

Both take the recall probability to be exogenous. In Katz (1986) there is a fixed probability $p$ that an unemployed worker, still waiting for his old job in period $t$, will be recalled in that period. An example could be an industry that faces two states of demand, high or low. The high demand state requires $H$ workers, the low demand state $L<H$ workers. Demand is currently low and $(H-L)$ workers are unemployed. The low demand state is either due to a transitory shock, or to a permanent decline in demand. If the shock is transitory the probability of a shift to high demand is generated from a Poisson process with a parameter $p$. This process generates an exogenous recall probability. Specifically, one unemployed workers's prospect of getting a recall does not depend on the decisions made by other unemployed workers.

Add a small, quite natural, twist to the example and this is no longer the case. Consider an industry, a sector or a region, that faces three demand states, high, medium or low. The number of workers required in each state are $H, M$ and $L$, respectively, where $H>M>L$. Demand has dropped from the high state to the low state, and there are $(H-L)$ unemployed workers. As above the low demand state is either due to a transitory shock, 
or to a permanent decline in demand. Everyone consent on this, but they also realize that even if we are in a transitory bad state, the market will never restore to old heights. The best one can hope for is a partial revival of demand. Specifically, if the shock is transitory there is a probability that demand will return to the medium level. That is, if demand revives $(M-L)$ workers will be rehired. Initially, there are $(H-L)$ unemployed individuals waiting for, at best, $(M-L)$ recalls. The probability of being recalled is no longer exogenous, but depends on the decisions made by other unemployed workers. One person's probability of being rehired in the injured sector, increases when another person decides to leave and search for a job elsewhere. A worker calculating private costs and benefits of the two alternatives, stay or move, will not take into account how his decision affects the prospects of other unemployed workers.

The aim of this paper is to understand how this externality slows down the transition process in an economy that encounters geographic or sectorial changes in economic activity. Particularly, we want to understand how different policy instruments, such as the replacement ratio of unemployment benefits, cash bonuses to workers that find a new job and government programs initiated to stimulate demand in the injured sector, affect the adjustment process.

In our model, situations arise where the number of workers that wait for a recall is higher than the number of possible job openings - which is consistent with the results of Meyer and Katz (1990). The fact that an excessive number of workers may be unemployed do not come as a surprise to search theorists; such patterns would be anticipated on the grounds of adverse incentives for job search created by, for instance, unemployment benefits. ${ }^{1}$ As noted by Meyer (1995), relating to unemployment insurance payments: "A prime cause of overpayments ... is the failure of claimants actively to seek work." In this paper, we explore another avenue that might lead to excessive unemployment, namely the positive externality associated with moving away from the injured sector. Hence, our model provides an additional argument for using policy measures to reduce unemployment. ${ }^{2}$ Obvious policy candidates are a reduction in the unemployment benefits (possibly politically unfeasible), or a moving cost subsidy. We show that both these reforms may have everything from no effect on unemployment, to instantly eradicating it. Although our set-up is highly stylized, the result non the less suggests that non-continuous responses to changes in policy variables may be expected.

\footnotetext{
${ }^{1}$ See for instance Burtless (1990) or Layard et al. (1994).

${ }^{2}$ Meyer (1995) provides an overview of U.S. experiments aimed at evaluating the effects of such reforms - most notably cash bonuses for quick reemployment, and job search programs.
} 
Another policy that might potentially reduce unemployment is job creation programs. ${ }^{3}$ This policy, however, turns out to be a double edged sword. Our analysis suggests, that job creation programs can slow down the structural adjustment process. This will be the case if the perceived recall probability is - at least in part - caused by the governments inability to commit not to initiate job a creation program. In such situations, we argue that the authorities may face a type Samaritan's Dilemma, as the expectations of job creation programs cause high levels of unemployment, which in turn may trigger the initiation of such programs. Also, we discuss the possibility that this 'game' between the authorities and the unemployed exhibits multiple equilibria.

In order to harness the effects of having an endogenous recall probability, as discussed above, we do not model search explicitly. Rather we assume that the unemployed can get a job in another sector with certainty if they pay a fixed moving cost, and hence we focus on the waiting decisions of workers. Thus, strictly speaking, we model a waiting game - a type of game that has been extensively studied within the literature on contests.

In contests two or more individuals exert costly effort to enhance their probability of winning a prize. The prize can be a seat in the parliament, a monopoly position, a high rank job in a firm, a patent etc. ${ }^{4}$ The waiting game is one subgroup within contests, most often referred to as the "war of attrition". In the classic version of the game, there are two players, each trying to wait the other out to win a prize. The game stops when one of the players concede, and the prize goes to the other player. In our example, the 'prize' would be getting a job without paying any moving costs. However, there are many workers $(H-L)$, competing for multiple 'prizes' $(M-L$ possible recalls). Two recent papers, Clark and Riis (1996) and Bulow and Klemperer (1999), extend the war of attrition game to situations where there is competition over more than one prize. We discuss their results and identify idiosyncrasies that prevent us from directly applying their findings. Our analysis then suggests an extension of the multiple prize war of attrition game.

The following section of our paper presents the formal model, with sections 3 and 4 discussing, respectively, the special cases of a constant and a declining probability of market revival. In section 5 we derive some policy implications, while section 6 addresses the links to the war of attrition

\footnotetext{
${ }^{3}$ This could be obtained through, for instance, increased subsidies to the afflicted industry, increased spending in the public sector etc.

${ }^{4}$ For an introduction to the theory of contests, see for instance Hirshleifer and Riley (1992). For more specialized applications, Lockard and Tullock (2001) (rent-seeking) and Lazear (1995) (labor market tournaments) can be recommended.
} 
literature. Section 7 concludes.

\section{The model}

We have already described the basic setting. One sector, denoted $A$, has experienced a slump in demand. Each unemployed worker can search for work elsewhere (sector $B$ ), or stay in sector $A$ and wait for better times.

If an unemployed worker moves she gets a job with certainty in another sector, but has to pay a moving $\operatorname{cost} c$. Of course, it would be more realistic to assume she has to search for a job elsewhere, and to let the outcome of this search process be uncertain. But, since it is the moving decision - not the search process - that concern us here, we simply assume that by paying a cost $c$, she gets a job in the other sector with certainty.

There are three different demand states facing the producers in this sector: a high demand state in which $H$ workers are needed, a low demand state in which $L$ workers are needed, and in between, a medium level in which $M$ workers are required in the sector. When we enter the economy, at time 0 , there has been a slump in demand and there are $H-L$ unemployed workers. The low demand state is either permanent or transitory. In the latter case there is a probability $p(t)$ that demand restores to a medium level in period $t$, conditional on demand being low in period $t-1$, where $t$ measures the number of periods that has passed since there was a drop in demand. If demand increases $(M-L)$ workers are rehired. ${ }^{5}$ If demand returns to the $M$ state, it will stay that way for ever.

An unemployed worker might leave sector $A$ immediately, at stage 0 , or wait one period. If she waits two outcomes are possible. Demand might partly recover, she then has a chance of being recalled to work in sector $A$. Alternatively, the $L$ state prevails and she faces the same move-wait decision in the next period. In general, an unemployed worker might wait $t$ periods and leave at stage $t>0$. Let $m_{t}$ be the number of workers that has left sector $A$ when we enter stage $t$.

To make life simple we assume there to be an institutionally set wage level $w$ in the economy. ${ }^{6}$ Unemployed workers receive unemployment benefits $b$

\footnotetext{
${ }^{5}$ We can relax this assumption and still bring home our basic argument. All we need, in order to get endogenous recall probabilities, is that there is some probability that the new permanent demand state is between the old high and the present low. It could for example be the case that if the low demand shock is transitory, demand either increases to its former high level or to a medium level.

${ }^{6}$ With a fixed wage level, a drop in demand in sector $A$ generate unemployment. If the wage was perfectly flexible, there would be no unemployment, but a drop in demand would lead to lower wages in sector $A$. Some of the workers might then prefer to leave
} 
per period. Each individual is risk neutral, has an infinite time horizon, a discount factor $\delta$, and seeks employment in another sector if expected lifetime earnings are higher elsewhere. Let $E\left[U(t)_{i}\right]$ denote the expected lifetime utility as seen from stage $t$ if decision $i=$ (move, wait) is taken. An unemployed worker moves at stage $t$ if $E\left[U(t)_{\text {move }}\right]>E\left[U(t)_{\text {wait }}\right]$. To get an interesting situation we assume that workers move from sector $A$ if the probability of a recall is zero, that is, we assume $w(1-\delta)^{-1}-c>b(1-\delta)^{-1}$. Hence, everyone who does not get a recall to a job in sector $A$ if demand increases, leaves sector $A$ immediately.

To characterize the transition process, that is, to characterize the sequence $\left\{m_{t}\right\}_{0}^{\infty}$, we need further assumptions about the probability that demand recovers to the medium level.

\section{Constant transition probability}

Assume first that the probability of a transition from state $L$ to state $M$ is constant over time, i.e. $p_{t}=p>0$ for all $t$. The recall probability need not be constant over time, however, since the probability of being recalled if demand increases, depends on the number of workers that has left sector $A$. Suppose demand is low at $t \geq 0$. If a person still waiting for better times in sector $A$, decides to wait for another period, she faces a recall probability of

$$
q_{t+1}=p \min \left\{\frac{M-L}{H-L-m_{t+1}}, 1\right\} .
$$

Note that the lowest possible recall probability is given by $\underline{q}=p\left(\frac{M-L}{H-L}\right)$, while the highest possible recall probability is equal to $p$.

We search for a transition pattern that constitutes a Nash-equilibrium at each stage. In other words, we require each individual's decision - stay or move - to be optimal given the decisions made by the others that are facing the same stay-move choice. To characterize such equilibrium patterns, we need to calculate the expected payoffs of the two alternatives, stay or move.

The discounted value of moving is independent of when a person moves; it is given by the difference between the discounted income and the moving costs: $E\left[U(t)_{\text {move }}\right]=\left(\frac{1}{1-\delta}\right) w-c$, for all $t \geq 0$.

The expected discounted value of waiting is given by

the sector. Assuming that the marginal productivity of labour decreases in the number of individuals working in sector $A$, we would still have a postitive externality associated with any exits from the sector. When one person moves from the sector, this increases the marginal productivity and the wage for those who choose to remain and wait for better times. 


$$
\begin{aligned}
E\left[U(t)_{\text {wait }}\right] & =b+\delta\left[q_{t+1} \frac{1}{1-\delta} w+\left(p-q_{t+1}\right)\left(\frac{1}{1-\delta} w-c\right)\right] \\
& +\delta(1-p)\left(\max \left\{E[U(t+1)]_{\text {wait }}, E[U(t+1)]_{\text {move }}\right\}\right)
\end{aligned}
$$

A worker who, at stage $t \geq 0$, decides to stay unemployed another period and wait for a job in sector $A$, earns unemployment benefits $b$ in the first period. At the start of the next period, the $M$ state is realized with probability $p$, and thus there is a probability $q_{t+1}$ that an unemployed worker is recalled to former work. If she is recalled she earns a wage $w$ in each period forever. With the complimentary probability, she is not recalled to work in her old sector. The probability that this happens is $p\left(1-\min \left\{\frac{M-L}{H-L-m_{t+1}}, 1\right\}\right)=p-q_{t+1}$. She then knows that there are no chances of getting recalled to work in sector $A$ in the future, and she moves immediately to seek work in sector $B$.

With probability $(1-p)$, demand stays at the low level, which means that the unemployed worker again faces the stay-move decision, and she chooses the option that maximizes her expected discounted earnings. Note that the individual value of waiting at time $t$ increases in the number of individuals that decide to move at stage $t$ as long as less than $H-M$ individuals have left up until and including time $t$. The reason is of course that the individual recall probability increases in $m_{t+1}$ if $m_{t+1}<H-M$.

\subsection{The equilibria}

We enter at stage 0 , immediately after there has been a decline in demand. There are three different exit patterns that are consistent with our equilibrium notion:

1. No one moves: $E\left[U(0)_{\text {wait }}\right]>E\left[U(0)_{\text {move }}\right]$ for $m_{1}=0 \Leftrightarrow q_{1}=\underline{q}$.

2. Some move, some stay: $E\left[U(0)_{\text {wait }}\right]=E\left[U(0)_{\text {move }}\right]$ for $0 \leq m_{1} \leq$ $H-M \Leftrightarrow q \leq q_{1} \leq p$.

3. Everyone moves: $E\left[U(0)_{\text {wait }}\right]<E\left[U(0)_{\text {move }}\right]$ for $m_{1}>H-M \Leftrightarrow q_{1}=$ $p$.

These three alternatives characterize different equilibrium patterns at stage 0 . Nothing happens in later periods as long as the $L$-state prevails: The exact same inequalities will hold at stage 1 - or at any period $t>0$ - as in period 0 , as long as demand is in the $L$-state, so no one finds it in their interest to move. For instance, if some, but not all of those who lost their 
job move at stage 0 , those left will be indifferent between moving or waiting also at later stages, and none of them will leave. To explain and verify these statements, and to characterize the equilibria in more detail, it is helpful to take hold of the interior equilibrium (case 2):

\subsection{The interior equilibrium}

In an interior equilibrium only a fraction of the unemployed leave, the rest decide to wait another period in sector $A$. For this to be an equilibrium, those waiting must get exactly the same expected lifetime income as those moving (otherwise some of them would move, or some of the movers would not). Formally, we have an interior equilibrium if and only if there exists an $m_{1}$, denoted $\widetilde{m}$, such that for $0 \leq \widetilde{m} \leq H-M$ we have $E\left[U(0)_{\text {wait }}\right]=$ $E\left[U(0)_{\text {move }}\right] .^{7}$ From equation 2 we see that $E\left[U(0)_{\text {wait }}\right]$ is a recursive value; it depends on the maximal value in the next period. We know that the value of moving is independent of when a person moves, hence, $E\left[U(t)_{\text {move }}\right]=$ $\left(\frac{1}{1-\delta}\right) w-c, \forall t$. Furthermore, we know that when $\widetilde{m}$ individuals leave at stage 0 , those left are indifferent between moving and staying at stage 0 . However, since the transition probability is constant over time, those waiting in sector $A$ are indifferent between moving and staying at stage 1 if and only if everyone who is left decides to stay. In other words, we know that the equilibrium will entail $E\left[U(1)_{\text {wait }}\right]=E\left[U(1)_{\text {move }}\right]=E\left[U(0)_{\text {wait }}\right]=E\left[U(0)_{\text {move }}\right]$ for $m_{2}=\widetilde{m}$. The same argument holds for any period where demand is still in the $L$-state..

We can use this information to find $\widetilde{m}$ :

$$
\begin{gathered}
E\left[U(0)_{\text {wait }}\right]=E\left[U(0)_{\text {move }}\right] \Longleftrightarrow \\
b+\delta p\left[\frac{M-L}{H-L-\widetilde{m}} \frac{1}{1-\delta} w+\left(1-\frac{M-L}{H-L-\widetilde{m}}\right)\left(\frac{1}{1-\delta} w-c\right)\right] \\
+\delta(1-p)\left(\frac{1}{1-\delta} w-c\right)=\left(\frac{1}{1-\delta}\right) w-c .
\end{gathered}
$$

\footnotetext{
${ }^{7}$ Strictly speaking, for this arbitrage condition to hold with equality, $m$ must be a continues variable. Since $m$ represents the number of individuals that move from sector $A$ it is not a continues variable. However, taking account of this whole number restriction does not change anything of importance, but it would make the exposition more cumbersome. Assume that $\widetilde{m}$ is not a whole number and let $\widetilde{m}_{-}$be the closest whole number lower than $\widetilde{m}$, and $\widetilde{m}_{+}$the closest whole number higher than $\widetilde{m}$. In the case of an interior equilibrium, instead of saying that we have an equilibrium $\widetilde{m}$ that makes those waiting indifferent between waiting and moving, we would have to say that those waiting get a slightly lower welfare than those moving if $\widetilde{m}_{-}$workers move, but a slightly higher welfare than those moving if $\widetilde{m}_{+}$workers move. Our Nash equilibrium concept then requires $\widetilde{m}_{+}$workers to move.
} 
Here, we have substituted $\left(\frac{1}{1-\delta}\right) w-c$ for $\max \left\{E[U(1)]_{\text {wait }}, E[U(1)]_{\text {move }}\right\}$ in (2). If we solve (3) we find

$$
\widetilde{m}=H-L-p \frac{c \delta}{(\delta c-c-b+w)}(M-L) .
$$

We know that $\widetilde{m}$ must satisfy $0 \leq \widetilde{m} \leq H-M$, which imply that there exists an interior solution if and only if

$$
1 \leq p \frac{c \delta}{(\delta-c-b+w)} \leq \frac{H-L}{M-L}
$$

This gives us sufficient information to characterize the transition process in this economy. Let $m_{1}^{*}$ be the equilibrium number of individuals that move from sector $A$ immediately after there has been slump in demand. Based on the discussion above we can conclude

Proposition 1 For a constant transition probability we have three types of equilibria:

1. $m_{1}^{*}=0$, for $p \frac{c \delta}{(c \delta-c-b+w)}>\frac{H-L}{M-L}$.

2. $m_{1}^{*}=\widetilde{m}=H-L-p \frac{\delta c}{\delta-c-b+w}(M-L)$, for $1 \leq p \frac{c \delta}{(c \delta-c-b+w)} \leq \frac{H-L}{M-L}$.

3. $m_{1}^{*}=H-L$, for $p \frac{c \delta}{(c \delta-c-b+w)}<1$

This proposition characterizes the number of unemployed workers that leave sector $A$ immediately after the drop in demand. ${ }^{8}$ Given a constant probability of a transition to state $M$, there will be no further action before demand recovers. If that happens, those who still do not get a job in the recovering sector, seek work elsewhere.

In the first case, the probability of market return, the unemployment benefits and the moving costs are all sufficiently high as to make no unemployed worker want to leave, even if this means that the recall probability $q$ is at its minimum, $\underline{q}$ (as it is when no one leaves).

In the second case, the prospects of waiting are slightly less appealing; if everyone else waits each person is better off moving. But if some workers leave the recall probability of those left increases, and for the quoted range of parameter values, it increases enough to compensate the remaining workers.

\footnotetext{
${ }^{8}$ We can think of this equilibrium as being produced by individuals leaving the sector sequentially. If workers differ by, say, having different moving costs, but the distribution of these costs are common knowledge, sequential moving will produce the same kind of equilibrium. However, with different moving costs, the individuals that leave have lower moving costs than the ones who stay.
} 
In the third case, the prospects in sector $A$ are really gloomy: Even if everyone else has left, and the recall probability is at its maximum, will an individual, still deliberating his options, not find it worthwhile to wait in sector $A$.

Two observations should be emphasized. There is an interesting discontinuity in the transition process described above. If the benefits of waiting are high, so high that we are in the interior of regime 1 where no one leaves, a small reduction in the benefits of waiting will not change the number of people leaving sector $A$. In regime 2 , where some choose to wait, there is a smooth response in the number of individuals that leave; if it becomes a little bit less attractive to wait in sector $A$, the response is a small increase in the number of people that leave. As we approach regime 3 , however, there is a dramatic change: A small reduction in the payoff of waiting, e.g. in the unemployment benefits, will now result in a surge of individuals leaving sector $A .^{9}$

The second observation worth mentioning is that if $M$ approaches $H$ the set of parameter values that gives an interior equilibrium shrinks. In the limit the interior equilibrium vanishes, in that case either none or all unemployed workers leave sector $A$.

\section{A decreasing transition probability}

In this section we study a case where the probability of a transition from state $L$ to $M$ decreases with time; $p_{t+1}<p_{t}$ for $t \geq 11^{10}$ Our aim is to characterize the flow of workers leaving sector $A$ as time passes after the slump in demand. Again, we focus on a transition process that satisfies a Nash equilibrium at each stage; candidate solutions must be such that the move or stay decision made by those still waiting in sector $A$ is optimal given the decision made by others.

In the preceding section we characterized three types of equilibria; (i) no unemployed workers move before the market returns (if it does), (ii) a fraction of the unemployed workers move at their first possible chance, the rest stays in sector $A$ until demand increases (if it does) and (iii) every unemployed worker moves immediately. The same type of exit patterns, where none, some

\footnotetext{
${ }^{9}$ If workers were not identical, the transition might be smoother.

${ }^{10} \mathrm{It}$ is, of course, the unemployed workers belief about how likely a transition is that determines their decision to move or not. Our specification, with a declining transition probability, can be thought of as a reduced form modelling of a situation where Bayesian updating of beliefs - given that the market has not yet returned - results in a lower estimate of the return probability as time passes.
} 
or all of the unemployed workers move, are equilibrium outcomes also with a decreasing transition probability. However, unless everyone moves from sector $A$ at once, there might be a steady flow of unemployed workers leaving the sector. Furthermore, the case where no one moves from sector $A$ might not be a stationary situation; eventually the probability of a transition to state $M$ could become sufficiently low as to induce at least some unemployed individuals to start leaving.

To characterize the transition patterns formally, let us again enter at stage 0 , immediately after there has been a decline in demand. If everyone moves, there can be no action afterwards. In the interior equilibrium, a fraction of the unemployed workers leave sector $A$ immediately, the rest wait. In the next period, if the market has not returned, the unemployed view the market return probability as being reduced; that is $p_{2}<p_{1}$. Then, if no more workers move, any single worker would benefit from moving (they were indifferent before; now the probability of market return is reduced). However, all workers might not exit sector $A$ : As some workers move, the recall probability is increased, compensating for the reduced market return probability. Thus, even at this stage, there might be an interior equilibrium, but it will entail a larger number of workers having left sector $A$.

Eventually, the probability of market return could become sufficiently low as to ensure that $m_{t}=H-M$. Then, again, the positive externality disappears, and a further decrease in the probability of market return induces every unemployed worker to leave the sector.

We again take hold of the interior equilibrium. In this equilibrium - for the workers that wait to be indifferent between staying and moving - a decreased market return probability has to be compensated for by an increased number of workers moving. In short, the recall probability has to be constant over periods. If it is not, our Nash equilibrium concept is violated: With a constant expected utility of moving, an increased recall probability invariably increases the relative payoff from staying. Then if workers were indifferent at a previous stage, they cannot be indifferent now (and vice versa). The same argument applies if the recall probability decreases over time.

Assume that the constant recall probability in the interior equilibrium is given by $a$; that is:

$$
p_{t+1} \frac{M-L}{H-L-m_{t+1}}=a .
$$

$a$ will be depending on the parameters of the model, $w, b, c$ and $\delta$. We determine the exact functional form of $a$ later.

The following figure represents (6) for $0 \leq m_{t+1} \leq H-M$. If the above equation specifies $m_{t+1}>H-M$, it is no longer valid, as discussed in the 
previous section. However, then the positive externality is no longer present, and a further reduction in the probability of market return means that every worker has an incentive to move. Thus for $p_{t+1}<a, m_{t+1}=H-L::^{11}$

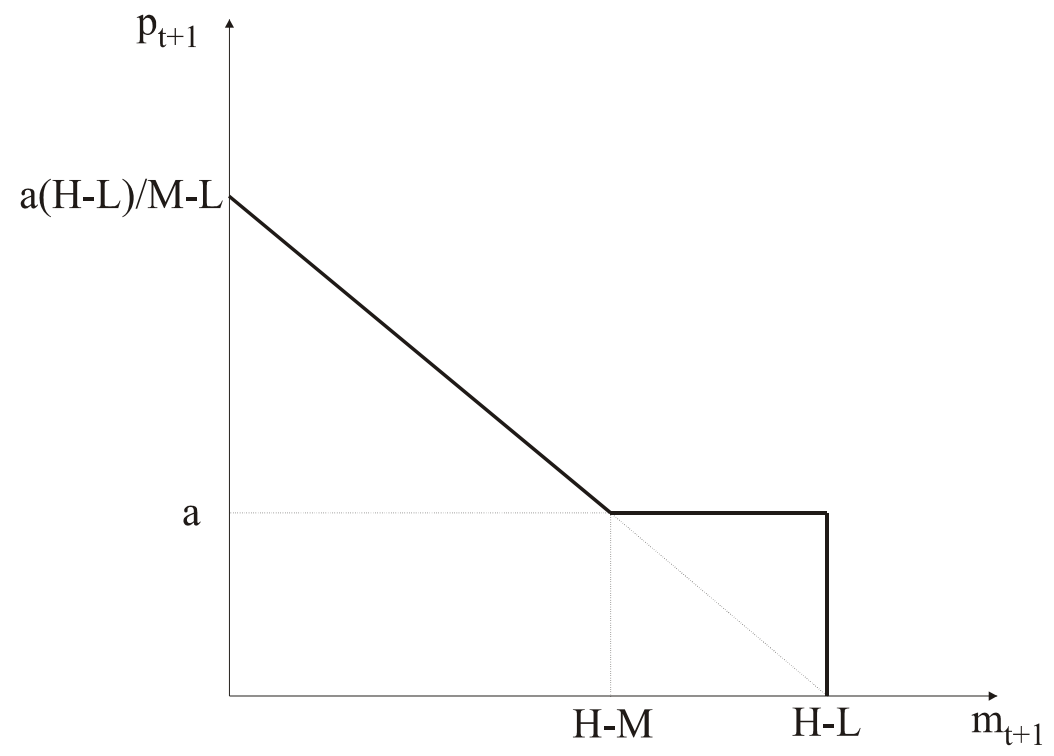

Figure 1

If $p_{t+1}$ lies between $a$ and $a \frac{H-L}{M-L}$, a reduction in $p_{t+1}$ will lead to more workers moving. As we can see, this rate of increase in the number of movers is constant for $p_{t+1} \in\left(a, a \frac{H-L}{M-L}\right)$. However, there is a discontinuous increase in the number of movers for $p_{t+1}=a$. This corresponds to the discussion in the last section, noting that $a$ will be dependent upon $w, b$ and $c .^{12}$

We will now find the explicit form of $a$. This is quite easy, since letting $p_{t+1}=p$ (a constant), we should be able to get exactly the same results as in the previous section. From the last section we had that every worker moved if and only if $p \frac{c \delta}{(c \delta-c-b+w)}<1 \Leftrightarrow p<\frac{w-b-c(1-\delta)}{c \delta}$. From figure 1, it is readily seen that the same will be true if and only if $p<a$. This is sufficient to determine $a$, which is then given by:

$$
a=\frac{w-b-c(1-\delta)}{c \delta}
$$

\footnotetext{
${ }^{11}$ The equilibria presented here can be obtained using a standard backward induction argument: With falling probability of market return, this probability will eventually reach zero or some non-zero constant value. Either way, the process effectively reaches a stationary state at some point in time. We are then in the exact same situation as in section 3, with a constant probability of market return. Using backward induction from this point in time, the payoff from staying is determined at each stage as a function of the outside option only (at each stage, the inside option has to be equal to the outside option). This uniquely determines the equilibrium presented here.

${ }^{12}$ Again, allowing for non-identical workers may smoothen the transition.
} 
Proposition 2 As long as the probability of market return is above some value $\bar{p}$, no workers move. For $p$ within a given interval $(p, \bar{p})$, some - but not all - workers move, and a decrease in the probability of market return results in a steady increase in the number of workers that move. However, for $p=\underline{p}$, there is a surge in the number of movers, and for $p<\underline{p}$, every unemployed worker has left sector 1 .

Proof. The results are evident from the above discussion provided $\bar{p}=$ $a \frac{H-L}{M-L}$ and $\underline{p}=a$.

One peculiarity of our model can be noted: In the general case, a reduction in the probability of market revival will (eventually) lead to more workers moving. As some workers move, the probability that the remaining workers get a job, provided the market revives, increases. However, when this probability reaches unity, and every remaining worker is assured a job provided market revival, then a further $\varepsilon$-reduction in the market revival probability induces every remaining worker to leave. Thus when recall (given market revival) finally is assured, all remaining workers move.

\section{$5 \quad$ Policy}

In regime 1 - where no one leaves sector $A$ - and in regime 2 - where some leave, but not all - there is unemployment in the economy. All, or some, of those who have lost their job in sector $A$, prefer to stay unemployed, even though there are available jobs elsewhere. The level of unemployment in period $t$ is simply given as

$$
\begin{aligned}
u_{t} & =H-L-m_{t} \\
& =\left\{\begin{array}{clc}
H-L & \text { if } & p_{t}>\bar{p} \\
(M-L) \frac{p_{t}}{a} & \text { if } & \underline{p} \leq p_{t} \leq \bar{p} \\
0 & \text { if } & p_{t}<\underline{p} .
\end{array}\right.
\end{aligned}
$$

We may distinguish between unemployment and excessive unemployment in this set up. It is perhaps beneficial, evaluated from society's point of view, to let some workers stay unemployed in $A$ and wait for better times, it depends on the shadow price of public funds (associated with unemployment transfers) and the magnitude of the moving costs. It is however never optimal - again evaluated from society's point of view - to let more workers wait than the maximum number of workers that can get rehired if market conditions improve. But in regime 1 and 2 this is exactly what happens, and hence, 
under the conditions specified in the propositions above, we get excessive unemployment.

Furthermore, if we consider the unemployed workers in sector $A$ as a group, they are obviously better off - again referring to regime 1 and 2 - if more workers leave sector $A$. The reason is of course the externality associated with moving from sector $A$; as long as fewer than $H-M$ workers have left, each person that decides to leave increases the recall probability of those waiting in sector $A$. Thus, our analysis provides an argument - augmenting the results of standard search theory - for using policy measures to reduce unemployment.

The level of unemployment in our model depends on various policy variables. By taking partial derivatives of the unemployment level $u_{t}$ in regime 1 and 2, with respect to the relevant policy variables, we find the following effects: In regime 1 no one leaves sector $A$ because they strictly prefer staying unemployed in $A$ over seeking employment elsewhere. A small change in any policy instruments have no effect on unemployment in this case. But, moving into regime 2, we find that a small reduction in unemployment benefits, or a small increase in a moving cost subsidy, means that more unemployed workers choose to leave sector $A$. Note that as we approach the boundary between regime 2 and 3 , a further reduction in unemployment benefits (or increase in the moving cost subsidy) generate a large response in the number of individuals that leave sector $A$ : If a policy change implies a shift from regime 2 to $3, M-L$ workers will leave sector $A$.

In the U.S., experiments have been conducted aimed at evaluating the effects of cash reemployment bonuses (see for instance Meyer (1995)). Such experiments seem to confirm that direct incentives do affect behavior of the unemployed. A reemployment bonus would in our simple model take the form of a moving cost subsidy. As we have discussed, such a subsidy will never increase unemployment, but wether it has a small or a large unemployment reducing effect depends on the recall expectations of the unemployed. However, as noted by Meyer (1995), permanent reemployment bonuses may prove to increase the number of unemployed, as the cost of leaving work is reduced with the introduction of such a bonus. This exact consideration cannot be captured in our simple set-up. However, in the following we discuss a related situation, where the initiation of government job creation programs may induce more workers to wait for a recall: 


\subsection{What if the government can affect the recall prob- ability?}

The government might want to initiate some sort of job creation program if unemployment is high. Modelling such a situation involves endogenizing the recall probability, which we have so far treated in a rather mechanical fashion. Expanding the set-up to include this kind of situation, however, only comes at a considerable expositional cost. For the purposes of this paper, we do not embark on such calculations as the most important insights can easily be grasped without explicitly modelling government behavior:

Say for instance that there is no chance that demand will recover if markets are left alone. In this case a positive recall probability stems from the fact that the government might initiate measures to reduce unemployment. As we have seen in the previous sections, such a recall probability will generally lead to fewer workers leaving (if the recall probability is not very small) than if the workers did not perceive recall to be a possibility. Thus the mere possibility of a policy intervention might in fact induce unemployment. We would expect these job creation programs to be costly to the government, and consequently, the authorities face a type Samaritan's Dilemma: If it was possible for the government to commit not to intervene, every worker would leave and there would be no unemployment. Without this commitment, however, more workers wait and structural adjustment slows down. The argument does not depend upon our extreme assumption that the market may not recover by itself. What is needed, is the possibility that the government albeit possibly involuntarily - contribute to the perceived revival probability.

If the government can affect the recall probability we might also get a situation with multiple equilibria. Assume for example that the government would not do anything to increase demand in sector $A$ if unemployment is relatively low. On the other hand, for high rates of unemployment, the government might feel forced to initiate job creation programs, and thus the Samaritan's Dilemma applies if only a limited number of workers move. It is not difficult to see that this kind of situation could lead to two equilibria: one where a lot of workers leave and the government does not intervene, and one where few workers leave and the government initiates job creation programs. Thus multiple equilibria might arise because the government cannot commit not to battle high levels of unemployment. 


\section{The war of attrition}

We have analyzed a "game" where many individuals contest over multiple 'prizes' ( $H-L$ laid off workers wait for $M-L$ possible recalls). As noted in the introduction, Clark and Riis (1996) and Bulow and Klemperer (1999) have discussed similar problems. In Bulow and Klemperer (1999) there is a war of attrition where $N+K$ firms compete for $N$ prizes. At each moment a firm, still hanging in there, can exit or continue the costly competition. Suppose the game ends when $K$ firms has left, and the payoff of a firm that opts out is independent of how long the game between those who remain lasts. In this case Bulow and Klemperer show that there exists only one symmetric equilibrium in which $K-1$ firms drop out immediately (or arbitrarily fast) and only $N+1$ firms remain: "If $N$ firms can be profitable in the market and dropouts pay no costs after exiting then competition in the symmetric equilibrium will immediately shake out to just one too many firms to be profitable." ${ }^{13}$ As the preceding analysis show, this result does not extend to our model. It is not, in general, the case that $H-M-1$ workers immediately move from sector $A$, leaving $M-L+1$ (one too many) workers waiting for a recall. A quick look at figure 1 tells us that this might happen in our model, but only as a special case.

The reason why the "one too many"-result does not apply in our setting is that "our" game ends with an exogenous probability: There is a positive probability that the market revives in each period, in that case the game is over and those waiting are randomly recalled to the new job openings. The game does, in other words, not come to an end when the number of individuals match the number of prizes, as in Bulow and Klemperer (1999). This is precisely why it might be the best response for a worker to stay unemployed in sector $A$ for one more period even though more than $M-L+1$ follow the same strategy. We can conclude that in a multiple prize war of attrition with uncertain termination, the shake-out might not be so extensive that only one too many participate in the game. In our model, less than $H-M-1$ workers leave if the exogenous probability that the game 'ends' soon is relatively high. However, if this probability is low, shake-out might be more extensive than in Bulow and Klemperer (1999).

13 "No costs after exiting" refers to costs that are incurred by an exiting firm after the firm has exited and before the game ends. A similar result is reported in Clark and Riis (1996); in a multi prize contest they find an equilibirum in which "one too many" actively participate in the contest (and they play a randomized strategy). 


\section{Conclusions}

In this paper we have studied worker reallocation when the unemployed face endogenous recall possibilities. We showed that structural adjustment might be slow - too slow even when taking the point of view of the workers themselves. Changing policy variables by a small amount can have no effect on unemployment if recall probabilities are high, but may have a huge impact if recall probabilities are sufficiently low. If the authorities can directly affect the recall probability, a problem resembling a Samaritan's Dilemma may arise: If the government cannot credibly commit to refrain from initiating job creation programs, more workers could choose to stay unemployed in anticipation of the program. Furthermore, this 'game' between the workers and the government may exhibit multiple equilibria.

The novel feature of our set-up is the modelling of recall probabilities as endogenous: When a worker chooses to leave the sector that has experienced a slump in demand, the remaining unemployed workers are all better off because the probability of being rehired, given market revival, increases. This positive externality leads there to be too few workers moving. Furthermore, it also creates the possibility that workers might not move all at once, instead dropping out of the sector in a continuous pace. Thus, in conclusion, too few workers leave, and the adjustment process drags out over time. On the surface, our analysis then augments the literature on search in suggesting the need for programs specifically targeted at inducing the unemployed to seek new employment. However, we show that such programs may - if not carefully designed - contribute to, rather than prevent, slow structural adjustment.

Finally, our model suggests an avenue for further research into the literature on contests. We argue that the set-up utilized in this paper is primarily a war of attrition with uncertainty concerning when the game ends. Comparing our results to those of Bulow and Klemperer (1999), we demonstrate how adding uncertainty in this way may have a non-trifling impact on the outcomes of such games.

\section{References}

[1] Bulow, J. and P. Klemperer (1999): "The generalized war of attrition", American Economic Review 89(1), 175-189.

[2] Burtless, Gary (1990): "Unemployment insurance and labor supply: A survey", In Unemployment Insurance, Hansen, W. L. and J. Byers eds., Madison, University of Wisconsin Press. 
[3] Clark, D. J. and C. Riis (1996): "A multi-winner nested rent-seeking contest", Public Choice 87(1-2), 177-184.

[4] Hirshleifer, J. and J. G. Riley (1992): The analytics of uncertainty and information, Cambridge Surveys of Economic Literature, Cambridge University Press.

[5] Katz, L. F. (1986): "Layoffs, recall and the duration of unemployment", NBER Working paper No.1825.

[6] Layard, R., Nickell, S. and R. Jackman (1994): The unemployment crises, London, Oxford University Press.

[7] Lazear, E. P. (1995): Personnel economics, Wicksell Lectures, MIT Press.

[8] Lockard, A. A. and G. Tullock (2001): Efficient rent-seeking: Chronicle of an intellectual quagmire, Lockard, A. A. and G. Tullock eds., Kluwer Academic 2001.

[9] Meyer, B. D. (1995): "Lessons from the U.S. unemployment insurance experiments", Journal of Economic Literature 33, 91-131.

[10] Meyer, B. D. and L. F. Katz (1990): "Unemployment insurance, recall expectations, and unemployment outcomes", Quarterly Journal of Economics 105(4), 973-1002.

[11] Pissarides, C. A. (1982): "Job search and the duration of layoff unemployment", Quarterly Journal of Economics 97(4), 595-612. 